\title{
Direct detection of ESKAPEc pathogens from whole blood with the use of the T2Bacteria Panel allows early antimicrobial stewardship intervention in patients with sepsis
}

Pavel Drevinek ${ }^{1}$, Jakub Hurych ${ }^{1}$, Milena Antuskova ${ }^{1}$, Jan Tkadlec ${ }^{1}$, Jan Berousek ${ }^{2}$, Zuzana Prikrylova $^{1}$, Jiri Bures ${ }^{1}$, Jaromir Vajter ${ }^{1}$, Martin Soucek ${ }^{1}$, Jan Masopust ${ }^{1}$, Vendula Martinkova $^{1}$, Jaroslava Adamkova ${ }^{1}$, Veronika Hysperska ${ }^{1}$, and Eliska Bebrova ${ }^{1}$

${ }^{1}$ Motol University Hospital

${ }^{2}$ Charles University

February 15, 2021

\begin{abstract}
In the microbiological diagnosis of bloodstream infections (BSI), blood culture (BC) is considered the gold standard test despite its limitations such as low sensitivity and slow turnaround time. A new FDA-cleared and CE-marked platform utilizing magnetic resonance to detect amplified DNA of the six most common and/or problematic BSI pathogens (Enterococcus faecium, Staphylococcus aureus, Klebsiella pneumoniae, Acinetobacter baumannii, Pseudomonas aeruginosa, and Escherichia coli) is available and may shorten the time to diagnosis and potentially improve antimicrobial utilization. Whole blood samples from hospitalized patients with clinical signs of sepsis were analyzed using the T2Bacteria Panel (T2Biosystems) and compared to simultaneously collected BC. Discrepant results were evaluated based on clinical infection criteria, combining supporting culture results and the opinion of treating physicians. A total of 55 samples from 53 patients were evaluated. The sensitivity and specificity of the T2Bacteria panel was $94 \%$ (16 out of 17 detections of T2Bacteria-targeted organisms) and 100\%, respectively, with $36.4 \%$ ( 8 of 22 ) causes of BSI detected only by this method. The T2Bacteria Panel detected pathogens on average 55 hours faster than standard BC. In our study, 9 of 15 patients with positive T2Bacteria Panel results received early-targeted antibiotic therapy, and/or their antimicrobial treatment was modified based on T2Bacteria Panel findings. Given the high reliability, faster time to detection, and easy workflow, the technique qualifies as a point of care testing approach.
\end{abstract}

Direct detection of Enterococcus faecium, Staphylococcus aureus, Klebsiella pneumoniae, Acinetobacter baumannii, Pseudomonas aeruginosa and Escherichia coli from whole blood with the use of the T2Bacteria Panel allows early antimicrobial stewardship intervention in patients with sepsis

Pavel Drevinek ${ }^{1 *}$, Jakub Hurych ${ }^{1}$, Milena Antuskova ${ }^{1}$, Jan Tkadlec ${ }^{1}$, Jan Berousek ${ }^{2}$, Zuzana Prikrylova ${ }^{2}$, Jiri Bures ${ }^{2}$, Jaromir Vajter ${ }^{2}$, Martin Soucek ${ }^{3}$, Jan Masopust ${ }^{3}$, Vendula Martinkova ${ }^{4}$, Jaroslava Adamkova ${ }^{4}$, Veronika Hysperska ${ }^{5}$, Eliska Bebrova ${ }^{1}$

1 Department of Medical Microbiology, $2^{\text {nd }}$ Faculty of Medicine, Charles University, Motol University Hospital, Prague, Czech Republic; pavel.drevinek@lfmotol.cuni.cz, jakub.hurych@lfmotol.cuni.cz, milena.antuskova@lfmotol.cuni.cz, jan.tkadlec@lfmotol.cuni.cz, eliska.bebrova@fnmotol.cz

2 Department of Anesthesiology and ICM, $2^{\text {nd }}$ Faculty of Medicine, Charles University, Motol University Hospital, Prague, Czech Republic; jan.berousek@fnmotol.cz, zuzana.prikrylova@fnmotol.cz, jiri.bures@fnmotol.cz, jaromir.vajter@fnmotol.cz 
${ }^{3}$ Department of Internal Medicine, $2^{\text {nd }}$ Faculty of Medicine, Charles University in Prague, Motol University Hospital, Prague, Czech Republic;martin.soucek@fnmotol.cz, jan.masopust@fnmotol.cz

${ }^{4} 3^{\text {rd }}$ Department of Surgery, $1^{\text {st }}$ Faculty of Medicine, Charles University, Motol University Hospital, Prague, Czech Republic; vendula.martinkova@fnmotol.cz, jaroslava.adamkova@fnmotol.cz

${ }^{5}$ Department of Rehabilitation and Sports Medicine, $2^{\text {nd }}$ Faculty of Medicine, Charles University, Motol University Hospital, Prague, Czech Republic; veronika.hysperska@fnmotol.cz

*Correspondence: pavel.drevinek@lfmotol.cuni.cz; Tel.: +42022443 5390

Abstract

In the microbiological diagnosis of bloodstream infections (BSI), blood culture (BC) is considered the gold standard test despite its limitations such as low sensitivity and slow turnaround time. A new FDA-cleared and CE-marked platform utilizing magnetic resonance to detect amplified DNA of the six most common and/or problematic BSI pathogens (Enterococcus faecium , Staphylococcus aureus ,Klebsiella pneumoniae, Acinetobacter baumannii ,Pseudomonas aeruginosa and Escherichia coli ) is available and may shorten the time to diagnosis and potentially improve antimicrobial utilization.

Whole blood samples from hospitalized patients with clinical signs of sepsis were analyzed using the T2Bacteria Panel (T2Biosystems) and compared to simultaneously collected BC. Discrepant results were evaluated based on clinical infection criteria, combining supporting culture results and opinion of treating physicians.

A total of 55 samples from 53 patients were evaluated. Sensitivity and specificity of T2Bacteria panel was 94\% (16 out of 17 detections of T2Bacteria-targeted organisms) and 100\%, respectively, with $36.4 \%$ (8 of 22) causes of BSI detected only by this method. The T2Bacteria Panel detected pathogens on average 55 hours faster than standard BC.

In our study, 9 of 15 patients with positive T2Bacteria Panel result received early-targeted antibiotic therapy and/or their antimicrobial treatment was modified based on T2Bacteria Panel findings. Given the high reliability, faster time to detection and easy workflow, the technique qualifies as a point of care testing approach.

Keywords: Bacteremia, Sepsis, T2MR, blood culture, rapid diagnostics, antimicrobial stewardship

\section{Introduction}

Bloodstream infections (BSIs) are characterized by high morbidity and mortality, consequences that have been linked to delays in early and accurate administration of antimicrobials [1].

Timely detection of BSI pathogens is an unmet need of today's medical microbiology practice. It remains largely dependent on blood culture (BC) [2] which performance is being continuously improved with new protocols [3] and new approaches for pathogen identification and determination of their antimicrobial susceptibility $[4,5]$. However, BC still has its inherent limitations including the requirements for large amounts of blood or the long turnaround time. Low sample volumes and exposure to antimicrobials prior to BC sample collection considerably increase the risk of false-negative BC results [6] . Despite these limitations, $\mathrm{BC}$ remains the gold standard and the first line tool for detecting BSIs.

In an effort to improve BSI management, several innovative diagnostic techniques have been developed to optimize direct pathogen detection from whole blood samples [6,7]. Some of them were introduced with limited success [8] or with inconclusive results of their validation studies [9, 10]. In this study we report our real-world experience with the T2Bacteria Panel (T2Biosystems, USA) which utilizes T2 magnetic resonance (T2MR) to detect six bacterial BSI pathogens (Enterococcus faecium, Staphylococcus aureus, Klebsiella pneumoniae, Acinetobacter baumannii, Pseudomonas aeruginosa andEscherichia coli, commonly referred to as ESKAPEc bacteria) directly from whole blood [11]. This culture-independent method can greatly shorten the time to positivity and like other existing rapid diagnostic methods, when integrated 
with an antibiotic stewardship program, may reduce the time to appropriate therapy and possibly improve infection-related outcomes such as duration of hospitalization or mortality.

2. Materials and Methods

Study population. This prospective study was conducted between November 2018 and April 2019 at Motol University Hospital, a tertiary care teaching hospital with over 2200 beds ( $18 \%$ of them being intensive care) and roughly 80,000 hospitalizations per year. Patients were eligible for study enrollment if they a) were being managed by Anesthesiology and Intensive Care Medicine Department or by intensive care units of Internal Medicine (including Hematology) or Surgery Departments, and b) presented clinical signs of BSI, evaluated at the discretion of the managing physician. Initially, only the patients whose samples were taken within standard opening hours of microbiology laboratory (weekdays from 6 am to $4 \mathrm{pm}$ ) were included to ensure immediate processing of blood samples. In order to intensify patient recruitment, eligibility hours in April 2019 were expanded from 6 am to midnight (except weekends).

Laboratory methods. All blood samples were subjected to both culture and the T2Bacteria Panel testing. BCs were processed using BACTEC FX Automated Blood Culture System (Becton Dickinson, USA). Positive flagged BCs were primed for Gram stain. Subsequent pathogen identification from positive BC bottles was accomplished with matrix-assisted laser desorption/ionization time-of flight mass spectrometry (Bruker Daltonics, USA) with the first attempt for identification made after 2 hours of pre-incubation on solid media [12].

Direct detection of six bacterial pathogens on the T2Dx instrument (T2Biosystems, USA) requires $4 \mathrm{~mL}$ whole blood specimens. During processing on the T2Dx, bacteria are concentrated directly in whole blood, then lysed to release the DNA. After amplification, target amplicon is hybridized with superparamagnetic particles and then detected by T2MR [13]. The results are available in as few as 3.5 hours. According to the manufacturer's instructions for use the T2Bacteria Panel is indicated as an aid in the diagnosis of bacteremia and results should be used in conjunction with other clinical and laboratory data.

All results were called to the managing clinician by the medical microbiologist. Results were reported as target detected or target not detected for each of the 6 bacterial targets and accompanied with the optional antimicrobial stewardship in case of positivity.

Study Endpoints and Statistical Analysis. The primary outcomes of this study were sensitivity and specificity of the T2Bacteria Panel, which were calculated using positive BC and/or T2Bacteria Panel results for a T2Bacteria-targeted organism as the reference. Species detected by BC but not included in the T2Bacteria Panel were excluded from sensitivity and specificity calculation. Similar to Nguyen et al.and De Angelis et al. , we developed infection criteria in order to resolve any discrepancies between T2Bacteria Panel and BC $[11,14]$. "Proven BSI" was defined as a positive BC (excluding potential BC contaminants) using a concurrently drawn specimen. T2Bacteria Panel results were determined to be "Probable BSIs" when the T2Bacteria-detected organism was isolated within 21 days from another BC specimen collected at a different time or from a clinical sample taken from another site (such as the abdominal fluid, urine, or bronchoalveolar lavage), indicating a plausible cause of infection. Finally, "Possible BSI" was defined as a negative BC, but a positive T2Bacteria Panel result in the absence of supporting culture data if the T2Bacteria Panel detected organism was a plausible cause of disease. Proven, probable and possible BSIs were included in final sensitivity and specificity calculation.

We also quantified the time to species identification between the BC and T2Bacteria Panel and noted any antibiotic stewardship interventions made as a result of the T2Bacteria Panel result. The time to species identification was defined as the hour interval between the time when a BC or T2Bacteria Panel sample was received in lab and the time when the ID results were reported. Continuous variables were presented as means and standard deviations, and were compared using a 2 sample t-test, as appropriate. Categorical variables were presented as counts and percentages, and were compared using an adjusted Wald confidence interval. Differences were considered to be statistically significant for $\mathrm{p}<0.05$. Statistical analyses were performed by using Minitab@19 Statistical Software (Minitab, LLC.). 


\section{Results}

During the study period, 55 samples were obtained and tested from 53 unique patient cases (Figure 1). Testing of blood samples by using T2Bacteria Panel resulted in the recovery of 16 positive detections from 15 samples yielding a $27 \%$ rate of positivity ( 15 of 55). All 16 detections were considered proven or probable cause of BSI based on the pre-specified definitions for BSI (see Methods). In total, 23 culture findings were recovered from $20 \mathrm{BC}$ samples. As 9 of them were deemed potential normal skin flora contaminants and not treated, only 14 findings from $14 \mathrm{BC}$ samples were considered to be clinically relevant. However, 5 of them were not identifiable using the T2Bacteria Panel (Figures 1 and 2) and thus excluded from sensitivity and specificity calculation. Since probable BSIs were assumed to be true positives that were missed by concurrent BC, sensitivity and positive predictive value was calculated to be $94 \%$ (95\% CI, 71.1\%-99.9\%) and $100 \%$ respectively (95\% CI, 82.9\%-100\%). Only one unexplained false-negative T2Bacteria detection (E. coli ) was identified in one sample that was T2Bacteria Panel-positive for another pathogen $(S$. aureus ). There were 40 concordant negative detections (in regard to T2Bacteria Panel targeted organisms), yielding a specificity of $100 \%$ (95\% CI, $92.4 \%-100 \%$ ) and a negative predictive value of $98 \%$ (95\% CI, $86.3 \%-99.9 \%$ ).

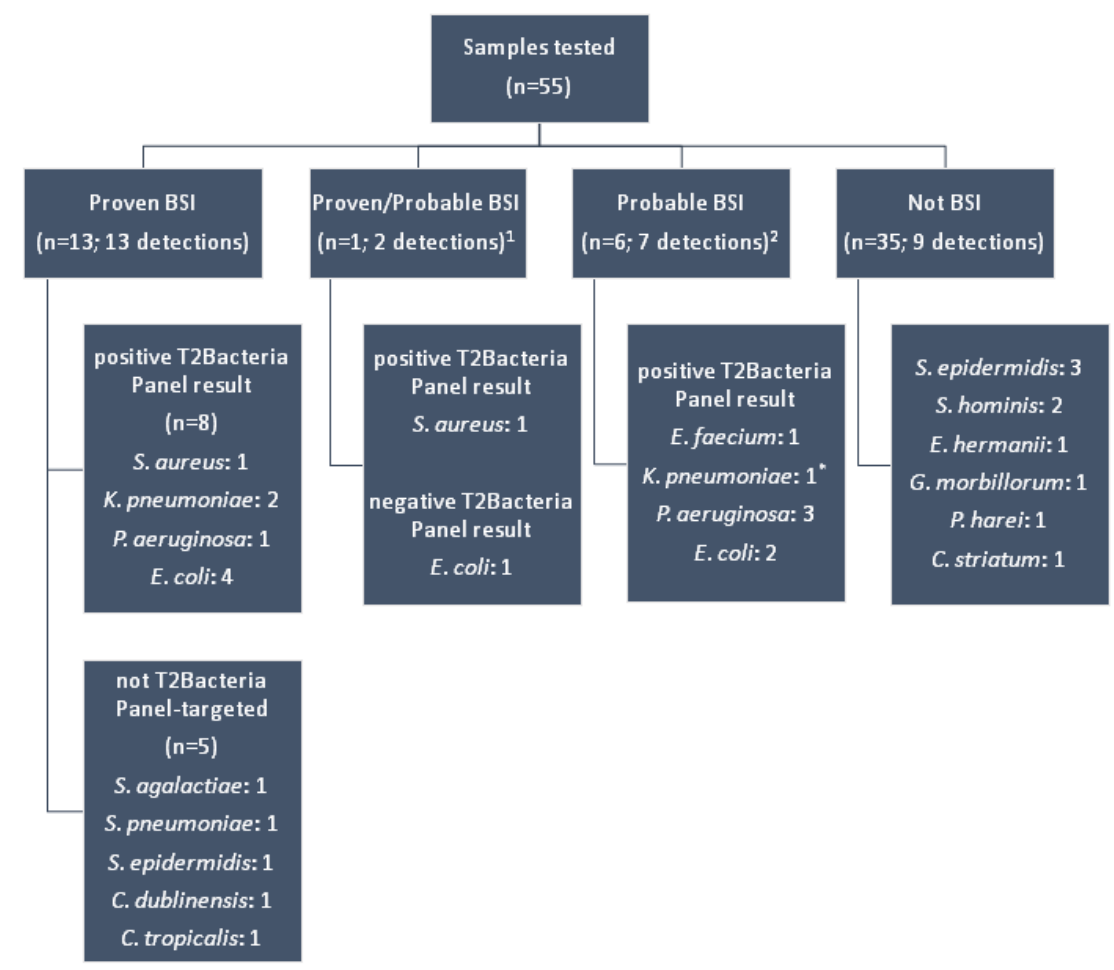

Figure 1. Performance of blood culture and the T2Bacteria Panel for diagnosis of BSI. ${ }^{1}$ In 1 sample, 1 T2Bacteria-targeted organism was identified by T2Bacteria Panel that was not identified in the paired blood culture. In this same sample, 1 T2Bacteria-targeted organism was identified in the paired blood culture, but not by the T2Bacteria Panel.

${ }^{2}$ In 1 sample, two T2Bacteria-targeted organisms were identified (K. pneumoniae and P. aeruginosa).

${ }^{*}$ Cross-reactivity between T2Bacteria K. pneumoniae channel and Klebsiella variicola was confirmed per the T2Bacteria Instructions For Use. 


\section{On-panel pathogen detections}

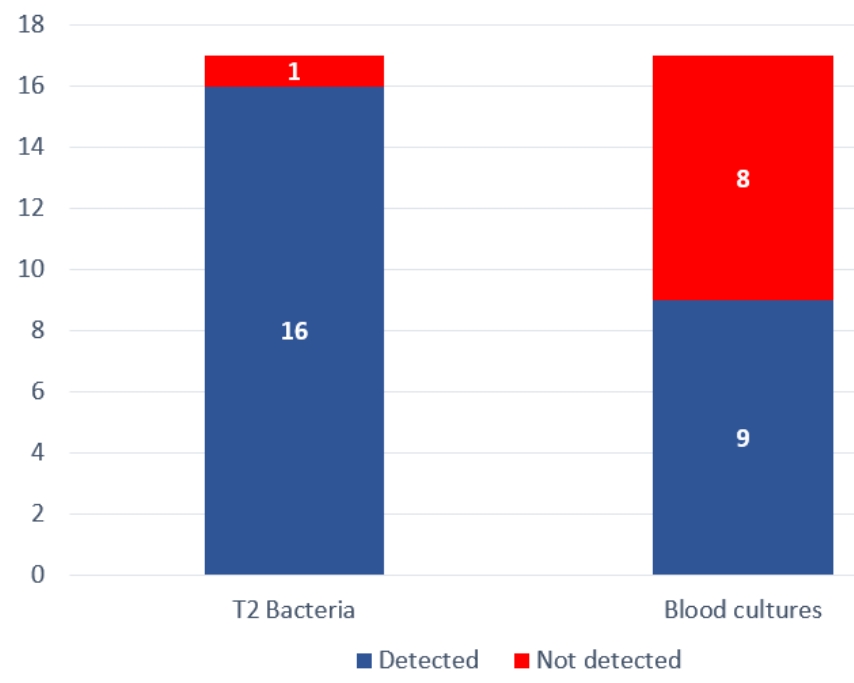

Figure 2. Comparison of on-panel pathogen detection (T2Bacteria vs. Blood cultures). 16 out of 17 proven and probable BSI's were detected by T2, whereas BC detected only 9 out of 17 .

The combined performance of $\mathrm{BC}$ and the T2Bacteria Panel for diagnosis of BSI is shown in Figure 3. Among all identified causes of proven and probable BSIs, 77\% (17 of 22) of positive detections were included on the T2Bacteria Panel. The most commonly identified species were E. coli and P. aeruginosa .

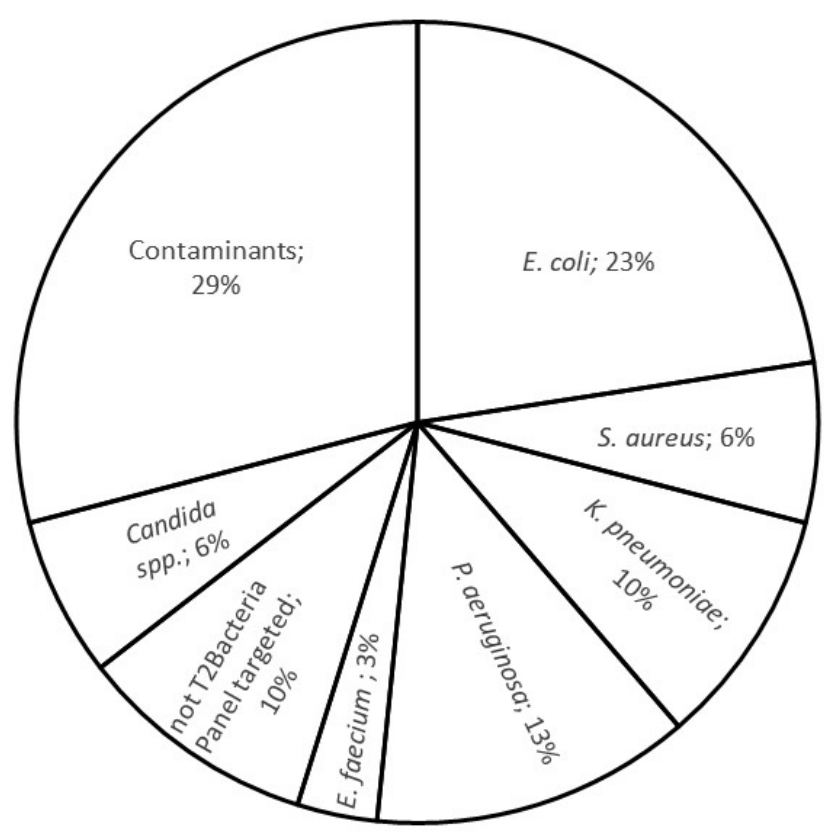

Figure 3. The distribution of all 31 positive detections comprising 15 different microbial species, detected 
by either method in 25 samples. A total of $55 \%$ findings were detectable with the T2Bacteria Panel. If contaminants are excluded, the T2Bacteria Panel covers $77 \%$ of clinically relevant findings.

The implementation of the T2Bacteria Panel decreased the time to species identification on average by 55 hours. The mean time from arrival to the laboratory to species identification was 6.1 hours $(\mathrm{SD} \pm 5.4$ ) for T2Bacteria Panel and 62 hours $(\mathrm{SD} \pm 54)$ for the conventional BC-based identification $(\mathrm{P}=0.001)$. The mean time from arrival to negative result was 7.4 hours $(\mathrm{SD} \pm 7)$ for T2Bacteria Panel and 119 hours $(\mathrm{SD} \pm 56)$ for the conventional $\mathrm{BC}(\mathrm{P}<0.001)$.

Rapid identification with the T2Bacteria Panel permitted early antimicrobial stewardship interventions related to faster time to detection and/or new finding of BSI pathogen and early initiation of targeted antibiotic therapy. Interventions were made in 9 of 15 patients with T2Bacteria Panel positive samples as seen on Table 1. T2Bacteria Panel negative results were not evaluated for this analysis.

\begin{tabular}{lllll}
\hline Patient sample ID & T2Bacteria Panel result & BC result & ATB change & ATB before T2Bacteria Panel result \\
\hline T2_30 & S. aureus & S. aureus & yes & amikacin \\
T2_20 & K. pneumoniae & K. pneumoniae & yes & imipenem \\
T2_25 & E. coli & E. coli & yes & piperacillin/tazobactam + metronid \\
T2_61 & E. coli & yes & cefotaxime + metronidazole \\
T2_1 & P. aeruginosa & yes & meropenem + vancomycin \\
T2_4 & K. pneumoniae, P. aeruginosa & negative & yes & cotrimoxazole + tigecycline \\
T2_7 & S. aureus & E. coli & yes & amoxicillin/clavulanic acid \\
T2_27 & P. aeruginosa & S. epidermidis & yes & amikacin \\
T2_46 & E. faecium & negative & yes & piperacillin/tazobactam \\
T2_47 & E. coli & E. coli & no & ceftriaxone \\
T2_48 & K. pneumoniae & K. pneumoniae & no & meropenem \\
T2_51 & E. coli & E. coli & no & cefotaxime + gentamicin \\
T2_22 & E. coli & negative & no & meropenem \\
T2_36 & E. coli & negative & no & meropenem + ciprofloxacin \\
T2_40 & P. aeruginosa & P. aeruginosa & no & meropenem \\
\hline
\end{tabular}

Table 1. Antimicrobial stewardship impact of positive T2Bacteria Panel results. Data from 15 patients with T2Bacteria Panel positive results. Please note that ATB treatment does not have to be T2Bacteria-species only directed, but it has to take into account also other microbiological findings and patient's clinical history (not reported in the Table). ATB=antibiotic.

\section{Discussion}

The timely administration of effective antimicrobial therapy is crucial for the survival of patients with sepsis [1]. Rapid diagnostic assays have been associated with improvements in time to appropriate antibiotic therapy by enhancing early identification of causative organisms for BSI $[4,5]$. Such data supports the coupling of rapid diagnostic technology with antimicrobial stewardship programs to optimize empirical antibiotic therapy and reduce time to targeted therapy.

In this study, we report the first interventional experience of the T2Bacteria Panel in the Czech Republic which identified the ESKAPEc pathogens directly from whole blood. Despite the limited number of bacterial species included on the T2Bacteria Panel, we found that $77 \%$ of all identified causes of proven and probable BSI (i.e., excluding common BC contaminants) were detected by the T2Bacteria Panel. High sensitivity of the T2Bacteria Panel was demonstrated by detection of 8 pathogens in 7 samples that were missed by BC, representing $36.4 \%$ ( 8 of 22) of the total number of identified causes of BSI. All of these confirmed true positives were from patients who were previously exposed to antibiotic therapy which is consistent with previous findings in patients with sepsis demonstrating that BC sensitivity is reduced by approximately $50 \%$ after the initiation of antimicrobial therapy [15]. It also suggests the T2Bacteria Panel performance 
may have limited interference from empirical antimicrobial treatment, which continues to be an issue with culture-based diagnostics [16].

The T2Bacterial Panel provided species identification in 6.1 hours on average, which was 55 hours faster compared to BC. These results are consistent with a multi-hospital survey demonstrating a median BC time to identification of 43.4 hours and replicates previous reports of T2Bacteria Panel's advantage of faster diagnosis compared to standard BC diagnostic methods [11, 14, 17, 18]. This allowed for early antibiotic stewardship interventions in $60 \%$ of our patients with T2Bacteria Panel positive samples.

This evaluation is limited by the small number of bacteremic patients who were enrolled in our single-center cohort study. We did not assess the potential role of the T2Bacteria-negative result on early therapeutic decisions, namely the early de-escalation of unnecessary, broad spectrum antibiotics. Our study did not assess the effect of the assay on patient outcomes, but it could be expected that faster time to effective antibiotic prescription would translate into reductions in length of stay and mortality based on previous reports that observed reductions in length of stay and mortality based on findings with alternative postculture molecular diagnostic methods [1, 19-22]. Lastly, the pathogen coverage by the T2Bacteria Panel is not inclusive of all causative organisms for BSI and cannot provide antimicrobial susceptibility information and is not intended to replace routine culture and susceptibility methods.

\section{Conclusions}

In this study, T2Bacteria Panel proved high sensitivity (by detecting 8 BC false-negative causes of BSI) and significant reduction of time to species identification (55 hours on average). A larger study should be conducted to determine the exact clinical impact of earlier T2Bacteria Panel results on length of hospital stay and mortality benefit in patients with BSI. However, based on our experience with rapid BSI diagnostics, T2Bacteria Panel represents the most promising currently available diagnostic tool. Implementation of the T2Bacteria Panel at our institution led to faster time to reliable detection of selected BSI pathogens and decreased time to administration of species-directed antibiotic therapy.

Author Contributions:Conceptualization, Pavel Drevinek, Eliska Bebrova, Jan Berousek, Martin Soucek, Vendula Martinkova, Veronika Hysperska; methodology, Pavel Drevinek, Jan Tkadlec, Jakub Hurych; software, Jakub Hurych; validation, Pavel Drevinek, Eliska Bebrova' ; formal analysis, Jakub Hurych; investigation, Milena Antuskova, Jakub Hurych; resources, Jan Berousek, Zuzana Prikrylova, Jiri Bures, Jaromir Vajter, Martin Soucek, Jan Masopust, Vendula Martinkova, Jaroslava Adamkova, Veronika Hysperska; data curation, Jakub Hurych, Pavel Drevinek with support of T2Biosystems analytics; writing - original draft preparation, Pavel Drevinek; writing - review and editing, Jakub Hurych, Jan Tkadlec; visualization, Pavel Drevinek with support of T2Biosystems analytics ; supervision, Eliska Bebrova; project administration, Jakub Hurych; funding acquisition, Pavel Drevinek. All authors have read and agreed to the published version of the manuscript.

Funding: This research was funded by the Ministry of Health of the Czech Republic: grant 15-28157A; conceptual development of research organization Motol University Hospital.

Acknowledgments: The authors acknowledge the contributions of T2Biosystems which provided the T2Dx instrument and data analysis support.

Conflicts of Interest: The authors declare no conflict of interest. The funders had no role in the design of the study; in the collection, analyses, or interpretation of data; in the writing of the manuscript, or in the decision to publish the results.

References

1. Kumar, A.; Roberts, D.; Wood, K.E.; Light, B.; Parrillo, J.E.; Sharma, S.; Suppes, R.; Feinstein, D.; Zanotti, S.; Taiberg, L.; Gurka, D.; Kumar, A.; Cheang, M. Duration of hypotension before initiation of effective antimicrobial therapy is the critical determinant of survival in human septic shock. Crit Care Med 2006 , $34,1589-1596$. 
2. Idelevich, E.A.; Seifert, H.; Sundqvist, M.; Scudeller, L.; Amit, S.; Balode, A.; Bilozor, A.; Drevinek, P.; Kocak Tufan, Z.; Koraqi, A.; Lamy, B.; Marekovic, I.; Miciuleviciene, J.; Muller Premru, M.; Pascual, A.; Pournaras, S.; Saegeman, V.; Schonheyder, H.C.; Schrenzel, J.; Strateva, T.; Tilley, R.; Wiersinga, W.J.; Zabicka, D.; Carmeli, Y.; Becker, K.; Escmid Study Group for Bloodstream Infections, E.; Sepsis. Microbiological diagnostics of bloodstream infections in Europe-an ESGBIES survey. Clin Microbiol Infect 2019, $25,1399-1407$.

3. Lamy, B.; Dargere, S.; Arendrup, M.C.; Parienti, J.J.; Tattevin, P. How to Optimize the Use of Blood Cultures for the Diagnosis of Bloodstream Infections? A State-of-the Art. Front Microbiol2016 , 7 , 697.

4. Bookstaver, P.B.; Nimmich, E.B.; Smith, T.J., 3rd; Justo, J.A.; Kohn, J.; Hammer, K.L.; Troficanto, C.; Albrecht, H.A.; Al-Hasan, M.N. Cumulative Effect of an Antimicrobial Stewardship and Rapid Diagnostic Testing Bundle on Early Streamlining of Antimicrobial Therapy in Gram-Negative Bloodstream Infections. Antimicrob Agents Chemother 2017 , 61, e00189-17.

5. Buehler, S.S.; Madison, B.; Snyder, S.R.; Derzon, J.H.; Cornish, N.E.; Saubolle, M.A.; Weissfeld, A.S.; Weinstein, M.P.; Liebow, E.B.; Wolk, D.M. Effectiveness of Practices To Increase Timeliness of Providing Targeted Therapy for Inpatients with Bloodstream Infections: a Laboratory Medicine Best Practices Systematic Review and Meta-analysis. Clin Microbiol Rev 2016 , 29 , 59-103.

6. Peker, N.; Couto, N.; Sinha, B.; Rossen, J.W. Diagnosis of bloodstream infections from positive blood cultures and directly from blood samples: recent developments in molecular approaches. Clin Microbiol Infect 2018, 24, 944-955.

7. Poole, S.; Kidd, S.P.; Saeed, K. A review of novel technologies and techniques associated with identification of bloodstream infection etiologies and rapid antimicrobial genotypic and quantitative phenotypic determination. Expert Rev Mol Diagn 2018 , 18 , 543-555.

8. Tkadlec, J.; Bebrova, E.; Berousek, J.; Vymazal, T.; Adamkova, J.; Martinkova, V.; Moser, C.; Florea, D.; Drevinek, P. Limited diagnostic possibilities for bloodstream infections with broad-range methods: A promising PCR/electrospray ionization-mass spectrometry platform is no longer available. Microbiologyopen 2020, 9 , e1007.

9. Warhurst, G.; Dunn, G.; Chadwick, P.; Blackwood, B.; McAuley, D.; Perkins, G.D.; McMullan, R.; Gates, S.; Bentley, A.; Young, D.; Carlson, G.L.; Dark, P. Rapid detection of health-care-associated bloodstream infection in critical care using multipathogen real-time polymerase chain reaction technology: a diagnostic accuracy study and systematic review. Health Technol Assess 2015 , 19 , 1-142.

10. Tkadlec, J.; Peckova, M.; Sramkova, L.; Rohn, V.; Jahoda, D.; Raszka, D.; Berousek, J.; Mosna, F.; Vymazal, T.; Kvapil, M.; Drevinek, P. The use of broad-range bacterial PCR in the diagnosis of infectious diseases: a prospective cohort study. Clin Microbiol Infect2019 , 25 , 747-752.

11. De Angelis, G.; Posteraro, B.; De Carolis, E.; Menchinelli, G.; Franceschi, F.; Tumbarello, M.; De Pascale, G.; Spanu, T.; Sanguinetti, M. T2Bacteria magnetic resonance assay for the rapid detection of ESKAPEc pathogens directly in whole blood. J Antimicrob Chemother2018, 73 , iv20-iv26.

12. Nunvar, J.; Drevinek, P. Identification of bacteria from aerobic and anaerobic blood cultures after short cultivation by matrix-assisted laser desorption/ionization-time-of-flight mass spectrometry. J Med Microbiol 2015, $64,1253-1255$.

13. Mylonakis, E.; Clancy, C.J.; Ostrosky-Zeichner, L.; Garey, K.W.; Alangaden, G.J.; Vazquez, J.A.; Groeger, J.S.; Judson, M.A.; Vinagre, Y.M.; Heard, S.O.; Zervou, F.N.; Zacharioudakis, I.M.; Kontoyiannis, D.P.; Pappas, P.G. T2 magnetic resonance assay for the rapid diagnosis of candidemia in whole blood: a clinical trial. Clin Infect Dis2015, 60 , 892-899.

14. Nguyen, M.H.; Clancy, C.J.; Pasculle, A.W.; Pappas, P.G.; Alangaden, G.; Pankey, G.A.; Schmitt, B.H.; Rasool, A.; Weinstein, M.P.; Widen, R.; Hernandez, D.R.; Wolk, D.M.; Walsh, T.J.; Perfect, J.R.; 
Wilson, M.N.; Mylonakis, E. Performance of the T2Bacteria Panel for Diagnosing Bloodstream Infections: A Diagnostic Accuracy Study. Ann Intern Med 2019, 170 , 845-852.

15. Cheng, M.P.; Stenstrom, R.; Paquette, K.; Stabler, S.N.; Akhter, M.; Davidson, A.C.; Gavric, M.; Lawandi, A.; Jinah, R.; Saeed, Z.; Demir, K.; Huang, K.; Mahpour, A.; Shamatutu, C.; Caya, C.; Troquet, J.M.; Clark, G.; Yansouni, C.P.; Sweet, D.; Investigators, F. Blood Culture Results Before and After Antimicrobial Administration in Patients With Severe Manifestations of Sepsis: A Diagnostic Study. Ann Intern Med 2019, 171, 547-554.

16. Farrell, J.J.; Hujer, A.M.; Sampath, R.; Bonomo, R.A. Salvage microbiology: opportunities and challenges in the detection of bacterial pathogens following initiation of antimicrobial treatment. Expert Rev Mol Diagn 2015 , 15 , 349-360.

17. Tabak, Y.P.; Vankeepuram, L.; Ye, G.; Jeffers, K.; Gupta, V.; Murray, P.R. Blood Culture Turnaround Time in U.S. Acute Care Hospitals and Implications for Laboratory Process Optimization. J Clin Microbiol 2018, 56 , e00500-18.

18. Voigt, C.; Silbert, S.; Widen, R.H.; Marturano, J.E.; Lowery, T.J.; Ashcraft, D.; Pankey, G. The T2Bacteria Assay Is a Sensitive and Rapid Detector of Bacteremia That Can Be Initiated in the Emergency Department and Has Potential to Favorably Influence Subsequent Therapy. J Emerg Med 2020 , 58 , 785-796.

19. Timbrook, T.T.; Morton, J.B.; McConeghy, K.W.; Caffrey, A.R.; Mylonakis, E.; LaPlante, K.L. The Effect of Molecular Rapid Diagnostic Testing on Clinical Outcomes in Bloodstream Infections: A Systematic Review and Meta-analysis. Clin Infect Dis $2017,64,15-23$.

20. Beganovic, M.; Costello, M.; Wieczorkiewicz, S.M. Effect of Matrix-Assisted Laser Desorption IonizationTime of Flight Mass Spectrometry (MALDI-TOF MS) Alone versus MALDI-TOF MS Combined with RealTime Antimicrobial Stewardship Interventions on Time to Optimal Antimicrobial Therapy in Patients with Positive Blood Cultures. J Clin Microbiol 2017 , 55 , 1437-1445.

21. Perez, K.K.; Olsen, R.J.; Musick, W.L.; Cernoch, P.L.; Davis, J.R.; Land, G.A.; Peterson, L.E.; Musser, J.M. Integrating rapid pathogen identification and antimicrobial stewardship significantly decreases hospital costs. Arch Pathol Lab Med 2013 , 137, 1247-1254.

22. Perez, K.K.; Olsen, R.J.; Musick, W.L.; Cernoch, P.L.; Davis, J.R.; Peterson, L.E.; Musser, J.M. Integrating rapid diagnostics and antimicrobial stewardship improves outcomes in patients with antibioticresistant Gram-negative bacteremia. J Infect 2014, 69 , 216-225. 